\title{
THE CORRELATION OF THE MAXIMA OF CORRELATED BROWNIAN MOTIONS
}

\author{
L. C. G. ROGERS, ${ }^{*}$ University of Cambridge \\ LARRY SHEPP, ${ }^{* *}$ Rutgers University
}

\begin{abstract}
We obtain an expression for the correlation of the maxima of two correlated Brownian motions.
\end{abstract}

Keywords: Brownian motion; correlation; Kantorovich-Lebedev transform

2000 Mathematics Subject Classification: Primary 60J65

\section{Introduction}

Finding the expectation (or the law) of a functional of a Brownian path is usually either quite straightforward (see [1] for examples of this type) or quite impossible; it is usually not too hard to guess into which category a particular case falls. However, the question which we deal with in this note is innocent to state, surprisingly tricky to answer, and falls somewhere between the two types of problem. This question arose naturally in an application to the estimation of the correlation between two stocks (see [6] for a full account), and can be simply stated as follows. Let $\left(W_{i}(t)\right)_{t \geq 0}, i=1,2$, be two standard Brownian motions with constant correlation $\rho$, where

$$
\mathrm{E}\left[W_{1}(s) W_{2}(t)\right]=\rho \min \{s, t\}, \quad \text { for all } s, t \geq 0,
$$

and let $S_{i}(t):=\sup _{s \leq t} W_{i}(s)$; what is $\mathrm{E}\left[S_{1}(t) S_{2}(t)\right]$ ?

Brownian scaling tells us that there must be some positive function $c:[-1,1] \rightarrow(0, \infty)$ such that

$$
\mathrm{E}\left[S_{1}(t) S_{2}(t)\right]=c(\rho) t
$$

so all we have to do is find $c$. The values $c(1)=1, c(0)=2 / \pi=0.6366198$, and $c(-1)=$ $2 \log (2)-1=0.3862994$ are known (for the latter case see, for example, [2]); they reduce to calculations for a single Brownian motion. The three values are not of course collinear, so the functional form of $c$ is not obviously trivial, but the departure from collinearity is not great:

$$
\frac{1}{2}\{c(-1)+c(1)\}-c(0)=0.0565274 .
$$

In this note, we shall derive the explicit form

$$
c(\rho)=\cos \alpha \int_{0}^{\infty} \mathrm{d} \nu \frac{\cosh v \alpha}{\sinh \nu \pi / 2} \tanh \nu \gamma
$$

for the function $c$, where $\rho=\sin \alpha, \alpha \in(-\pi / 2, \pi / 2)$, and $2 \gamma=\alpha+\pi / 2$.

Received 22 February 2006; revision received 8 June 2006.

* Postal address: Statistical Laboratory, Centre for Mathematical Sciences, University of Cambridge, Wilberforce Road, Cambridge CB3 0WB, UK. Email address: 1.c.g.rogers@statslab.cam.ac.uk

** Postal address: Department of Statistics, Rutgers University, Piscataway, NJ 08854-8019, USA.

Email address: shepp@stat.rutgers.edu 


\section{Calculating $c$}

We begin with some notation. We write

$$
g:=\frac{1}{2}\left\{D_{1}^{2}+2 \rho D_{1} D_{2}+D_{2}^{2}\right\}
$$

for the infinitesimal generator of $W$, where $D_{i}:=\partial / \partial x_{i}$. We write

$$
X_{i}(t):=S_{i}(t)-W_{i}(t), \quad i=1,2,
$$

for the process of the heights below the maxima, which is a correlated two-dimensional Brownian motion in $\mathbb{R}_{+}^{2}$ with normal reflection on the axes. Though the values of $X_{i}(0)$ are both zero in this application, we allow ourselves to consider the process starting from different points of $\mathbb{R}_{+}^{2}$, as is customary in Markov process theory. We write $T$ for an exponential $(\lambda)$ variable independent of $W$, and $\operatorname{set} \theta:=\sqrt{2 \lambda}$.

We break the calculation into a sequence of goals, each a consequence of the next, until we finally arrive at a goal we can attain.

Goal 1. Calculate

$$
\begin{aligned}
f\left(x_{1}, x_{2}\right) & :=\mathrm{P}\left[x_{1} \leq S_{1}(T), x_{2} \leq S_{2}(T)\right] \\
& =\int_{0}^{\infty} \lambda \mathrm{e}^{-\lambda t} \mathrm{P}\left[x_{1} \leq S_{1}(t), x_{2} \leq S_{2}(t)\right] \mathrm{d} t .
\end{aligned}
$$

Attaining Goal 1 is equivalent to solving the problem, because we then obtain

$$
\lambda^{-1} c(\rho)=\int_{0}^{\infty} \int_{0}^{\infty} f\left(x_{1}, x_{2}\right) \mathrm{d} x_{1} \mathrm{~d} x_{2} .
$$

To achieve Goal 1, we aim for Goal 2.

Goal 2. Calculate

$$
\begin{aligned}
\tilde{f}\left(x_{1}, x_{2}\right): & =\mathrm{P}\left[S_{1}(T) \leq x_{1}, S_{2}(T) \leq x_{2}\right] \\
& =\mathrm{P}\left[\tau>T \mid X_{1}(0)=x_{1}, X_{2}(0)=x_{2}\right],
\end{aligned}
$$

where $\tau=\inf \left\{t: X_{1}(t) X_{2}(t)=0\right\}$.

Goal 2 gives us Goal 1 because

$$
\begin{aligned}
1-f\left(x_{1}, x_{2}\right) & =\mathrm{P}\left[S_{1}(T) \leq x_{1}\right]+\mathrm{P}\left[S_{2}(T) \leq x_{2}\right]-\tilde{f}\left(x_{1}, x_{2}\right) \\
& =1-\mathrm{e}^{-\theta x_{1}}+1-\mathrm{e}^{-\theta x_{2}}-\tilde{f}\left(x_{1}, x_{2}\right) .
\end{aligned}
$$

Now,

$$
\begin{aligned}
\hat{f} & :=1-\tilde{f}\left(x_{1}, x_{2}\right) \\
& =\mathrm{P}\left[\tau<T \mid X_{1}(0)=x_{1}, X_{2}(0)=x_{2}\right] \\
& =\mathrm{E}\left[\mathrm{e}^{-\lambda \tau} \mid X_{1}(0)=x_{1}, X_{2}(0)=x_{2}\right]
\end{aligned}
$$

will clearly satisfy

$$
(\lambda-g) \hat{f}=0
$$


with boundary conditions $\hat{f}=1$ on the axes. Using this, we see from (2) that

$$
f\left(x_{1}, x_{2}\right)=\mathrm{e}^{-\theta x_{1}}+\mathrm{e}^{-\theta x_{2}}-\hat{f}\left(x_{1}, x_{2}\right)
$$

must solve

$$
(\lambda-g) f=0
$$

with boundary conditions

$$
\begin{aligned}
& f\left(x_{1}, 0\right)=\mathrm{e}^{-\theta x_{1}}, \\
& f\left(0, x_{2}\right)=\mathrm{e}^{-\theta x_{2}} .
\end{aligned}
$$

We now present our next goal.

Goal 3. Solve the partial differential equation (PDE) given by (3), (4), and (5).

For Goal 3, we transform the state variables as follows, where $\rho=\sin \alpha$ :

$$
X_{t}:=X_{1}(t) \sec \alpha-X_{2}(t) \tan \alpha, \quad Y_{t}=X_{2}(t) .
$$

As is easily confirmed, the process $Z_{t}:=X_{t}+\mathrm{i} Y_{t}$ is now a complex Brownian motion in the wedge

$$
\Omega_{\rho}:=\left\{r \mathrm{e}^{\mathrm{i} \varphi}: r \geq 0,0 \leq \varphi \leq 2 \gamma\right\},
$$

where

$$
2 \gamma=\alpha+\frac{\pi}{2}
$$

The Brownian motion $Z$ experiences skew reflection on the boundary of $\Omega_{\rho}$ in the direction $(-\sin \alpha, \cos \alpha)$ on $\mathbb{R}_{+}$and in the direction $(1,0)$ on the other side of the wedge.

Remark. Brownian motion in the wedge with skew reflection was studied in [7], where criteria were given for the corner of the wedge to be visited and for there to be possible escape from the corner; see also [5] for a brisk summary of the results. The criterion in [7] leads to the (initially surprising) conclusion that if $\rho>0$ then the corner of the wedge will be visited. In terms of $W$, this says that there will be times $t$ such that

$$
W_{1}(t)=S_{1}(t) \quad \text { and } \quad W_{2}(t)=S_{2}(t)
$$

a property that would certainly not be satisfied if the Brownian motions were independent.

Writing $h(x+\mathrm{i} y)=f\left(x_{1}, x_{2}\right)$, we therefore find that $h$ satisfies the PDE

$$
\left(\lambda-\frac{1}{2} \Delta\right) h=0
$$

with the boundary condition

$$
h\left(r e^{i \varphi}\right)=\exp (-\theta r \cos \alpha)
$$

for $\varphi=0$ or $\varphi=2 \gamma$. Writing the Laplacian in polar coordinates, we obtain the PDE

$$
\left(\theta^{2}-D_{r r}-\frac{1}{r} D_{r}-\frac{1}{r^{2}} D_{\varphi \varphi}\right) h=0,
$$


for $h$, where (for example) $D_{r r}:=\partial^{2} / \partial r^{2}$. Now the PDE (7) has separable solutions of the form

$$
h_{v}\left(r \mathrm{e}^{\mathrm{i} \varphi}\right)=K_{\mathrm{i} v}(\theta r) \cosh (v(\varphi-\gamma)),
$$

for $v>0$, in terms of the usual Bessel functions $K_{\beta}$; the key is to combine these using Kantorovich-Lebedev transforms, a technique the authors learned from Henry McKean (see $[3$, p. 150]). We claim that the integral combination

$$
h\left(r \mathrm{e}^{\mathrm{i} \varphi}\right)=\frac{2}{\pi} \int_{0}^{\infty} \cosh (\nu \alpha) \frac{\cosh v(\varphi-\gamma)}{\cosh v \gamma} K_{\mathrm{i} v}(\theta r) \mathrm{d} \nu
$$

is the solution to the PDE (7) with the required boundary conditions (6). The fact that this solves the PDE follows from that fact that it is a linear combination of separable solutions and, to confirm the boundary behaviour, we quote the identity

$$
\frac{2}{\pi} \int_{0}^{\infty} K_{\mathrm{i} v}(r) \cosh v \alpha \mathrm{d} v=\exp (-r \cos \alpha),
$$

for $0 \leq \alpha \leq \pi / 2$ (see [4, p. 244]).

The expression (8) achieves Goal 3; hence, Goal 2 and finally Goal 1 are achieved. To obtain the constant $c(\rho)$ we just have to integrate the solution $h$ over the domain $\Omega_{\rho}$, not forgetting the (constant) Jacobian. We find that

$$
\begin{aligned}
\lambda^{-1} c(\rho) & =\cos \alpha \int_{0}^{\infty} r \mathrm{~d} r \int_{0}^{2 \gamma} \mathrm{d} \varphi \int_{0}^{\infty} \mathrm{d} \nu \frac{2}{\pi} \cosh (\nu \alpha) \frac{\cosh \nu(\varphi-\gamma)}{\cosh \nu \gamma} K_{\mathrm{i} v}(\theta r) \\
& =\frac{\cos \alpha}{\theta^{2}} \int_{0}^{2 \gamma} \mathrm{d} \varphi \int_{0}^{\infty} \mathrm{d} \nu \frac{\cosh \nu \alpha}{\sinh \nu \pi / 2} \frac{\cosh \nu(\varphi-\gamma)}{\cosh \nu \gamma} \\
& =\frac{\cos \alpha}{\theta^{2}} \int_{0}^{\infty} \mathrm{d} \nu \frac{\cosh \nu \alpha}{\sinh \nu \pi / 2} 2 \tanh \nu \gamma .
\end{aligned}
$$

The integration taking us from the first line of this display to the second is from $[8, \mathrm{p} .388$, Equation (8)]. Finally, we explicitly have

$$
c(\rho)=\cos \alpha \int_{0}^{\infty} \mathrm{d} \nu \frac{\cosh \nu \alpha}{\sinh \nu \pi / 2} \tanh \nu \gamma,
$$

as stated in (1).

\section{References}

[1] Borodin, A. N. And Salminen, P. (2002). Handbook of Brownian Motion - Facts and Formulae, 2nd edn. Birkhäuser, Basel.

[2] Garman, M. B. and Klass, M. J. (1980). On the estimation of security price volatilities from historical data. J. Business 53, 67-78.

[3] Lebedev, N. N. (1972). Special Functions and Their Applications. Dover, New York.

[4] Oberhettinger, F. (1972). Tables of Bessel Transforms. Springer, New York.

[5] Rogers, L. C. G. (1989). A guided tour through excursions. Bull. London Math. Soc. 21, 305-341.

[6] Rogers, L. C. G. And Zhou, F. (2006). Estimating correlation from high, low, opening and closing prices. Tech. Rep., Statistical Laboratory, University of Cambridge.

[7] Varadhan, S. R. S. and Williams, R. J. (1985). Brownian motion in a wedge with oblique reflection. Commun. Pure Appl. Math. 38, 405-443.

[8] Watson, G. N. (1966). A Treatise on the Theory of Bessel Functions. Cambridge University Press. 